\title{
Anisotropic corner diffusion as origin for dendritic growth on hexagonal substrates
}

\author{
Harald Brune ${ }^{\text {a, }}$, Holger Röder ${ }^{\text {a }}$, Karsten Bromann ${ }^{\text {a }}$, Klaus Kern ${ }^{\text {a }}$, \\ Joachim Jacobsen $^{\mathrm{b}}$, Per Stoltze ${ }^{\mathrm{b}}$, Karsten Jacobsen ${ }^{\mathrm{b}}$, Jens Nørskov ${ }^{\mathrm{b}}$ \\ a Institut de Physique Expérimentale, EPF Lausanne, CH-1015 Lausanne, Switzerland \\ ${ }^{b}$ Center for Atomic-scale Materials Physics and Physics Department, Technical University of Denmark, DK-2800 Lyngby, Denmark
}

Received 1 November 1995; accepted for publication 8 December 1995

\begin{abstract}
$\mathrm{Ag}$ aggregation on $\mathrm{Ag}(111), \mathrm{Pt}(111)$, and $1 \mathrm{ML} \mathrm{Ag}$ pseudomorphically grown on $\mathrm{Pt}(111)$, has been studied with variable temperature STM. These systems all have in common that dendritic patterns with trigonal symmetry rather than randomly ramified aggregates, which would be expected for a simple hit and stick mechanism, form. Dendrites are characterized by preferential growth in the $\langle\overline{1} \overline{1} 2\rangle$-directions, i.e., perpendicular to A-steps. The key process for their formation has been found to be diffusion of one-fold corner atoms towards neighboring steps. Calculations with the effective medium theory show that this relaxation is highly asymmetric with respect to the two different kinds of close-packed steps. It leads to dendritic growth as verified by kinetic Monte-Carlo simulations which agree well with experiment.
\end{abstract}

Keywords: Computer simulations; Epitaxy; Growth; Low index single crystal surfaces; Nucleation; Platinum; Scanning tunneling microscopy; Silver; Surface diffusion

In the "classical" diffusion limited aggregation (DLA) computer models, clusters are formed as randomly diffusing particles sticking irreversibly to the perimeter of a growing aggregate [1,2]. These models always produce randomly ramified aggregates, no matter whether they are carried out on a lattice or not (off-lattice DLA) [3]. Nonequilibrium aggregation processes in nature, however, quite often result in dendritic patterns which are characterized by preferred growth directions [4,5]. Despite a consider-

\footnotetext{
* Corresponding author. Fax: +41 21693 3604; E-mail: harald.brune@ipe.dp.epfl.ch.
}

able theoretical effort, the relationship between ordered and randomly ramified patterns is not yet solved [6,7]. Both objects have a fractal dimension close to $1.7[8,9]$, but their different shape is evident for the unaided eye (compare e.g. Fig. 4A and 4C). It is generally accepted that the key to dendritic growth is anisotropy [10]. This can either be linked to the symmetry of the aggregating particles themselves, which is then amplified to the overall pattern shape as the aggregate grows (a well known example for this is the snowflake), or it can be due to the symmetry of the underlying substrate. Examples for this latter case are given in Fig. 1, where we observe dendritic growth for two-dimensional metal aggregation on three hexagonally close-packed substrates. 

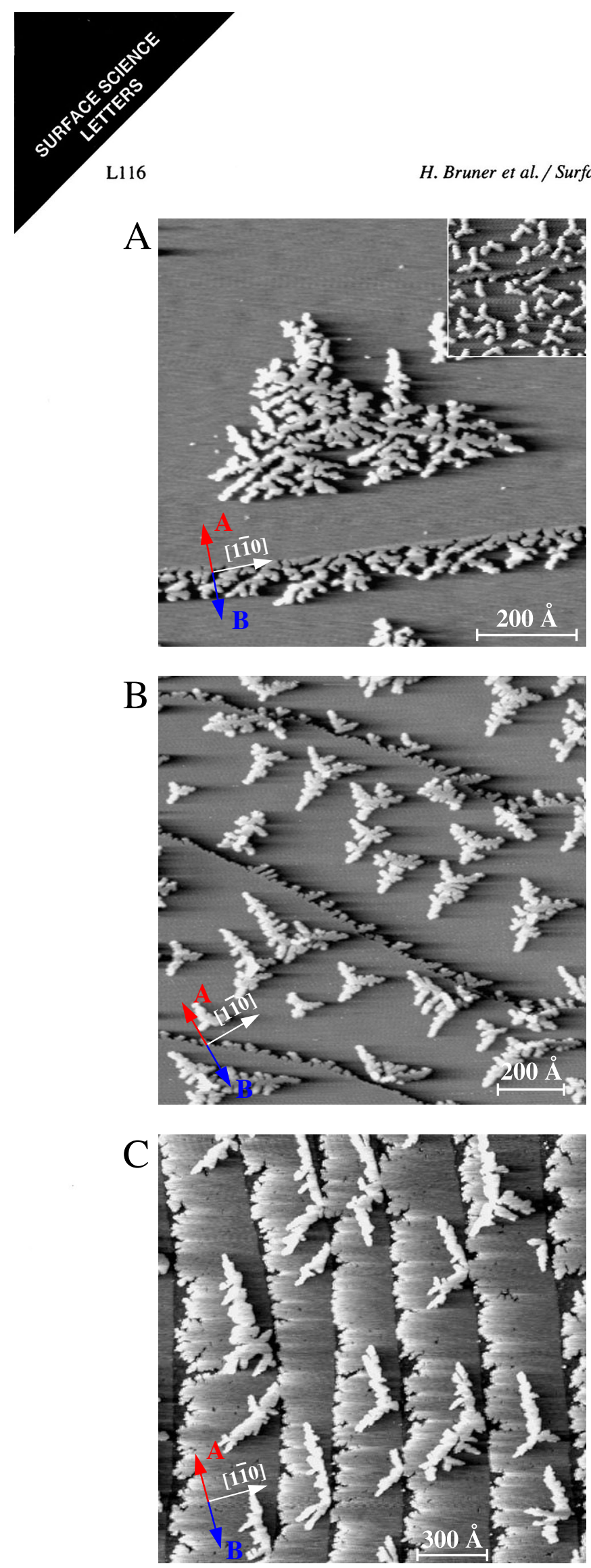

In order to reproduce dendritic structures basically two approaches have been followed to modify the hit and stick mechanism in DLA models. One is the introduction of curvature dependent sticking probabilities [11] and the second noise reduction, where atoms are only attached if the approached site has been visited $m-1$ times before [12]. Both mechanisms are, however, irrelevant for low temperature metal epitaxy, since there the sticking probability is 1 and independent of curvature.

Already in the first metal deposition experiments watched with the STM (at $300 \mathrm{~K}$ ), ramified growth and irregular island shapes were reported [13-15]. In particular, the Au aggregates on $\mathrm{Ru}(0001)$ have been considered as an example for classical DLA clusters because of their fractal dimension [15]. However, it had been realized later that their branch width (100$200 \AA$ ) points to a considerable amount of edge diffusion [16-19]. Similarly, the first results reported for lower temperature, showing ramified islands for homoepitaxy on $\mathrm{Pt}(111)$, were also interpreted as the physical realization of the hit and stick mechanism [20].

The first time dendrites have been reported, and their distinction to randomly ramified aggregates was made clear, was for the aggregation of $\mathrm{Ag}$ on $\mathrm{Pt}(111)$ $[9,21]$. These results have inspired kinetic MonteCarlo simulations which reproduced the randomly ramified islands obtained in the experiment at very low deposition flux [22]. Since they assume a hit and stick mechanism and neglect the specific substrate symmetry (which both holds for aggregation of much bigger clusters [23]) they were unable to simulate dendritic growth. This also holds for those models that study metal nucleation on a square lattice [2426]. On the other hand, simulations performed on a hexagonal lattice either focus on the shape of compact islands on Pt $[27,28]$, or they do not distinguish between the two types of close-packed steps present on a hexagonal substrate $[16,29]$. There are close-

Fig. 1. Dendritic patterns form for deposition of $\mathrm{Ag}$ onto $\mathrm{Pt}(111)$ at $130 \mathrm{~K}(\mathrm{~A})$ and $80 \mathrm{~K}$ (inset), onto $\mathrm{Ag}(111)$ at $110 \mathrm{~K}(\mathrm{~B})$, and on a pseudomorphic monolayer of $\mathrm{Ag}$ adsorbed on $\mathrm{Pt}(111)$ at $170 \mathrm{~K}$ (C). The STM topographs have been recorded isothermally to deposition and show the surface as it appears when illuminated from the left. The Ag coverage is $\Theta=0.12 \mathrm{ML}$, the deposition flux $F=1.1 \times 10^{-3} \mathrm{ML} \mathrm{s}^{-1}$. 


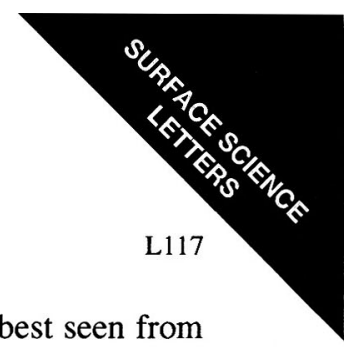

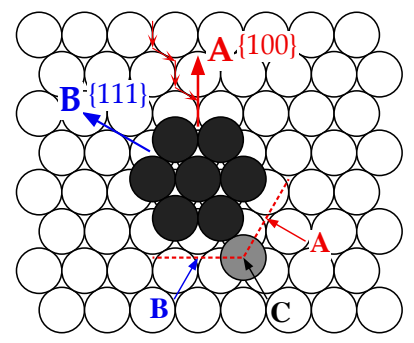

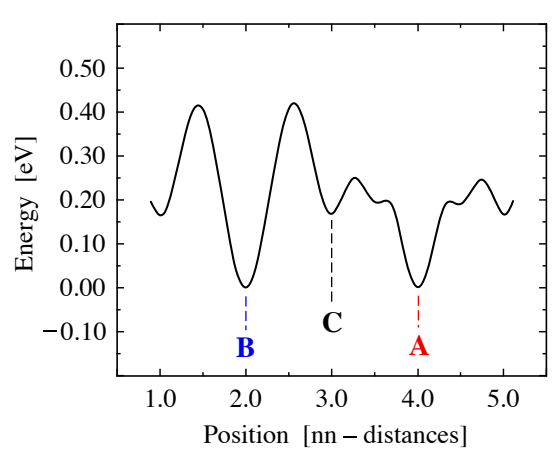

Fig. 2. Difference in total energy of an $\mathrm{Ag}$ adatom diffusing around a Ag heptamer on Pt(111) as calculated with EMT (A- and B-directions are indicated in the ball model). The diffusion processes with the lowest barriers evidently are that from a corner (C) and the hcp site close to it to an A-step.

packed $\{111\}$-facets and more open $\{100\}$-facets (see model in Fig. 2), which have been called B- and A-steps, respectively [30].

Thus, to date, a realistic description of the atomic processes leading to dendritic growth in metal aggregation on hexagonal substrates was lacking. We will present here a microscopic model explaining dendrite formation and discuss the importance of the different relaxation processes at the island edge and their influence on the aggregate shape.

Patterns formed by the aggregation of $\mathrm{Ag}$ on three hexagonally close-packed metal surfaces, i.e. the (111) surfaces of $\mathrm{Pt}, \mathrm{Ag}$, and one $\mathrm{Ag}$ monolayer pseudomorphically adsorbed on $\mathrm{Pt}(111)$ [31], all have in common a clear dendritic shape (Fig. 1). The variable temperature STM images (for experimental setup see e.g. Ref. [32]) show that their branches preferentially grow into three directions, which are rotated by $120^{\circ}$ with respect to each other. They are the crystallographic $\langle\overline{1} 12\rangle$-directions which are perpendicular to A-steps, one of them has been labeled A. For the first case of $\mathrm{Ag} / \mathrm{Pt}(111)$ (Fig. 1A) the trigonal symmetry of the aggregate is best seen from its triangular envelope, but also the longest central branches clearly point into the preferred growth directions. Notice also that the material attached to the straight substrate step in the lower part of the image does not grow perpendicular to the edge (which would be the B-direction since this step is a $\{111\}$ facet, i.e. of B-type) but instead in two of the A-directions forming angles of $\pm 30^{\circ}$ with respect to the step. At $80 \mathrm{~K}$ islands are too small to branch more than once and the growth anisotropy leads to equally oriented Y's (inset Fig. 1A). For Ag/Ag(111) (Fig. 1B) the preferred growth in three directions is clearly seen from the central branches of the aggregates. The trigonal symmetry of the aggregates on one pseudomorphic Ag layer is even more pronounced. Their shape resembles very much that of needle crystals, which are the extreme case of anisotropic growth [33]. These examples strongly suggest that dendritic growth is common for low temperature metal aggregation on hexagonally close-packed metal surfaces. In fact also Pt islands formed at low $T$ have dendritic shapes with preferential growth in A-direction [34].

In order to analyze the atomistic process responsible for the formation of dendrites on hexagonal surfaces, we will distinguish between two diffusion processes that can take place at the island edge. Depending on whether an adatom starts from a site which is laterally two- or one-fold coordinated to the island, i.e. whether the initial site is an edge or a corner, we will call its displacement edge or corner diffusion, respectively [35]. For corner diffusion the final coordination can be 1 or 2 . For edge diffusion the final coordination is 2 . The case where an edge atom (2-fold coordinated) goes to a corner (1-fold) becomes important at higher temperatures, and should be treated separately.

It turns out that this distinction is rather useful since these processes have quite different effects on the aggregate's shape. Edge diffusion discussion leads to a thickening of the aggregates' branches [16-19]. Closer inspection of the dendrites (Fig. 1) indeed reveals that the branches become thicker in going from images (A) to (C) (notice the different scale). From the narrow branches for $\mathrm{Ag}$ on $\mathrm{Pt}(111)$ ( $2 \pm 1$ atoms wide) in Fig. 1A we can deduce that edge diffusion is practically frozen (at $130 \mathrm{~K}$ and the 
applied deposition flux) [19]. Edge diffusion is active for $\mathrm{Ag} / \mathrm{Ag}(111)$ at $110 \mathrm{~K}$ since branches are $8 \pm 1.5$ atoms wide (Fig. 1B) [19]. It is even more involved in the case of $\mathrm{Ag} / 1 \mathrm{ML} \mathrm{Ag} / \mathrm{Pt}(111)$ leading to $18 \pm 3$ atoms wide branches (Fig. 1C). From the fact that dendritic growth occurs in all examples shown in Fig. 1, independent from the amount to which edge diffusion is involved, we can argue that it is very unlikely to be the origin of dendritic growth [36].

In order to get insight into the relaxation processes at the aggregate perimeter we have calculated diffusion barriers with the effective medium theory (EMT) [37]. The results show that for all three cases where we found dendritic growth in the STM experiment, corner diffusion to an A-step has a much lower barrier than displacement from a corner to a B-step (Fig. 2 and Table 1). This asymmetry can be rationalized from simple geometric reasons. From inspection of the model (Fig. 2) it becomes evident that displacement from a corner to an A-step can be done via an hcp-hollow site without loosing the coordination to the heptamer, whereas for diffusion towards a B-step the hcp site is situated too close towards the island. Thus the adatom has to walk almost over an on-top site, which is much more costly in energy.

For some systems like $\mathrm{Pt}(111)$ [28] and $\mathrm{Au}(111)$ homoepitaxy, the corner asymmetry is less pronounced in the EMT calculations and the direction is even inverted, i.e., displacement to B-steps is slightly preferred (Pt: $E_{\mathrm{Ac}} / E_{\mathrm{Bc}}=212 \mathrm{meV} / 173 \mathrm{meV}$, Au: $\left.E_{\mathrm{Ac}} / E_{\mathrm{Bc}}=146 \mathrm{meV} / 101 \mathrm{meV}\right)$. This inversion is due to tensile stress inherent in these systems leading to a strong contraction at the island edge, which facilitates the diffusion from corner to B-steps. Easier diffusion to B-steps similarly is expected for adsorbates with a significantly smaller lattice constant than the substrate.

It is therefore important to notice that there is a second anisotropy which generally holds for hexagonally close-packed surfaces. An atom that diffuses towards an A-step close to a corner does this via the hcp site located between the two fcc sites at the corner and the A-step (ball model Fig. 2). Already at this point, it feels the two-fold coordination at the step and has a much lower barrier to go there than to diffuse to the corner site (see asymmetry in activation energy around the hcp site and the flashes indicating an approaching atom in Fig. 2). On a B-step, the decision whether the atom goes to the corner or the step is made much earlier so that it is not guided to the step.

For $\mathrm{Ag}$ aggregation, both these asymmetries give a significant preference in populating A-steps. It is important to note that both diffusion processes, i.e., corner to A-step and that from the hcp site to an A-step, have an activation energy comparable to, or even below, that obtained for terrace diffusion. Therefore, relaxation towards A-steps is active as soon as nucleation and aggregation set in, and the classical hit and stick DLA clusters do not form. The barriers for the more difficult corner process (corner to B step), and those for edge diffusion, as well as to escape from an edge to a corner (see Table 1), are significantly higher, which implies that these processes can be frozen completely at low temperatures.

Let us now see how this specific preference for A-steps decides on the shape of the growing aggre-

Table 1

Energy barriers for the most relevant atomic diffusion processes involved in metal aggregation on hexagonal substrates for the systems studied in Fig. 1 ( $E_{\mathrm{m}}$ terrace diffusion, $E_{\mathrm{Ac}}$ corner to A-step, $E_{\mathrm{Bc}}$ corner to B-step, $E_{\mathrm{Ae}}$ and $E_{\mathrm{Be}}$ A- and B-edge diffusion, $E_{\mathrm{Aec}}$ and $E_{\mathrm{Bec}}$ denote diffusion from A- and B-edges to corners); the experimental values for terrace diffusion have been obtained by the analysis of the saturation island density as a function of $\mathrm{T}$ at a critical cluster size of 1 [39]

\begin{tabular}{lllllll}
\hline $\begin{array}{l}\text { Ag diffusion } \\
\text { on substrates }\end{array}$ & $\begin{array}{l}E_{\mathrm{m}}[\mathrm{meV}] \\
\mathrm{STM}\end{array}$ & $\begin{array}{l}E_{\mathrm{m}}[\mathrm{meV}] \\
\mathrm{EMT}\end{array}$ & $\begin{array}{l}E_{\mathrm{Ac}} / E_{\mathrm{Bc}} \\
\mathrm{EMT}\end{array}$ & $\begin{array}{l}E_{\mathrm{Ae}} / E_{\mathrm{Be}} \\
\mathrm{EMT}\end{array}$ & $\begin{array}{l}E_{\mathrm{Aec}} / E_{\mathrm{Bec}} \\
\mathrm{EMT}\end{array}$ & $\begin{array}{l}E_{\mathrm{Ac}} / E_{\mathrm{Bc}} \\
\mathrm{KMC}\end{array}$ \\
\hline $\mathrm{Pt}(111)$ & $157 \pm 10$ & 80 & $80 / 248$ & $187 / 389$ & $244 / 415$ & $\begin{array}{l}E_{\mathrm{m}}[\mathrm{meV}] \\
\mathrm{KMC}\end{array}$ \\
& & & & & $160 / 500$ \\
$\mathrm{Ag}(111)$ & $97 \pm 10$ & 67 & $73 / 139$ & $222 / 300$ & $260 / 330$ & - \\
$1 \mathrm{ML} \mathrm{Ag} / \mathrm{Pt}(111)$ & $60 \pm 10$ & 50 & $39 / 165$ & $167 / 354$ & $231 / 356$ & - \\
\hline
\end{tabular}

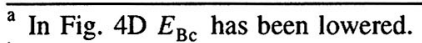

${ }^{\mathrm{b}} E_{\mathrm{m}}$ has been lowered for $130 \mathrm{~K}$ in order to account for dimer instability at this temperature, see text). 


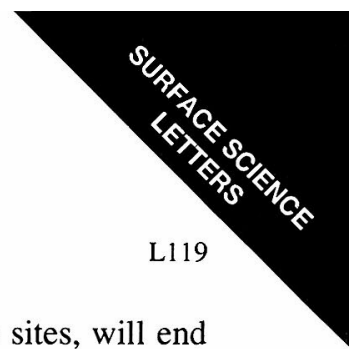

gate in a growth scenario (Fig. 3A). For symmetry reasons we start from a tetramer which is bound by two A- and B-steps. Atoms impinging at corners adjacent to an A-step, as well as those approaching the A-step at the two neighboring hcp sites, will end up at the A-step, which significantly increases the probability of attachment there. Once the atoms are at A-steps, they form a protruding tip (e.g. atom 1 in

(A)

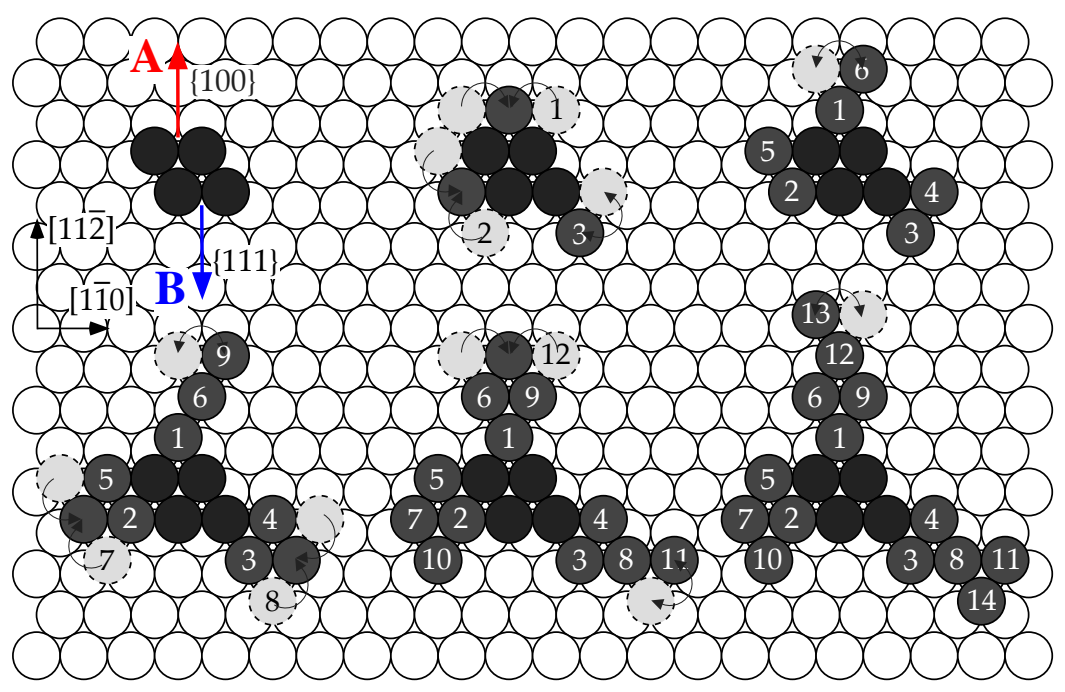

(B)

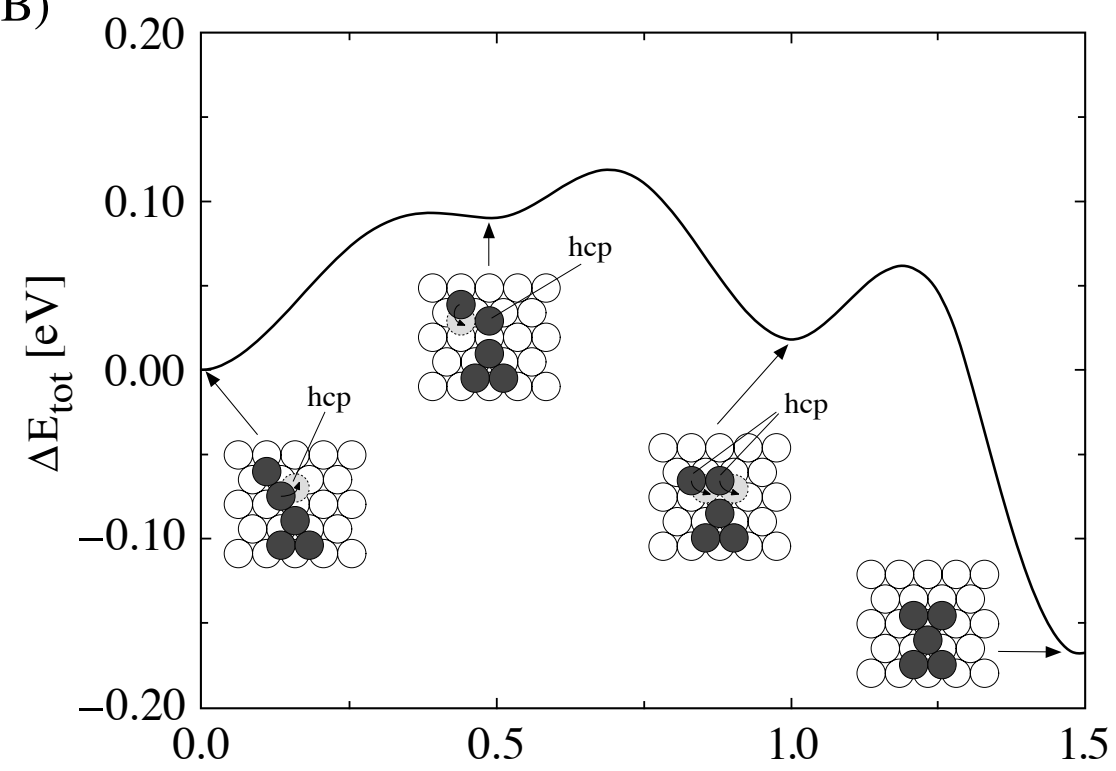

Fig. 3. Scenario showing how dendritic Y's with branches in the A-direction grow on a hexagonal surface if corner diffusion towards A-steps is active while that towards B-steps is frozen (A). Movement from the possible initial sites of impingement (light gray) leading to the same final adsorption site have been marked by bent arrows. The energy diagram in (B) shows the instability of atomic chains via a collective relaxation of both atoms. 


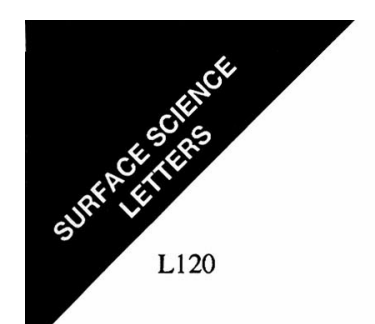

Fig. 3A) and thus have a slightly higher capture rate than straight steps (this is known as "tip effect" or Mullins Sekerka instability [2,38]). Atoms arriving at such an atomic "tip", can diffuse back and forth between the two corner sites but they cannot escape from there, since this would be diffusion to the adjacent B-steps, which is frozen. If a second atom arrives at the respective free corner, a new A-step is
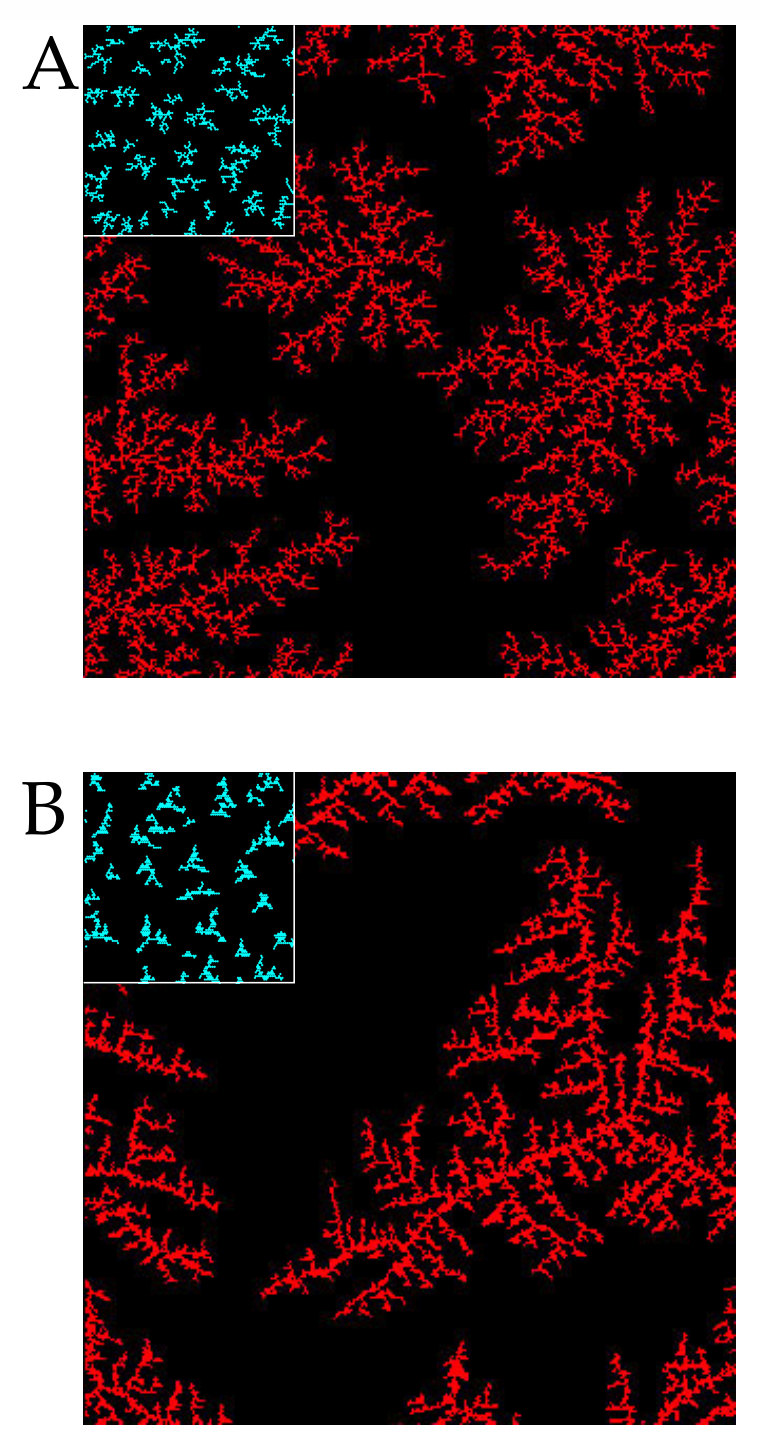

formed, which then again collects very effectively diffusing atoms and growth in the A-direction proceeds. If the atom arrives such that a linear chain in the close-packed direction would be formed (atoms 1, 6, and 9), the EMT calculations suggest (Fig. 3B) that these chains can relax with a low energy barrier to a more compact form which also ends with an A-step.
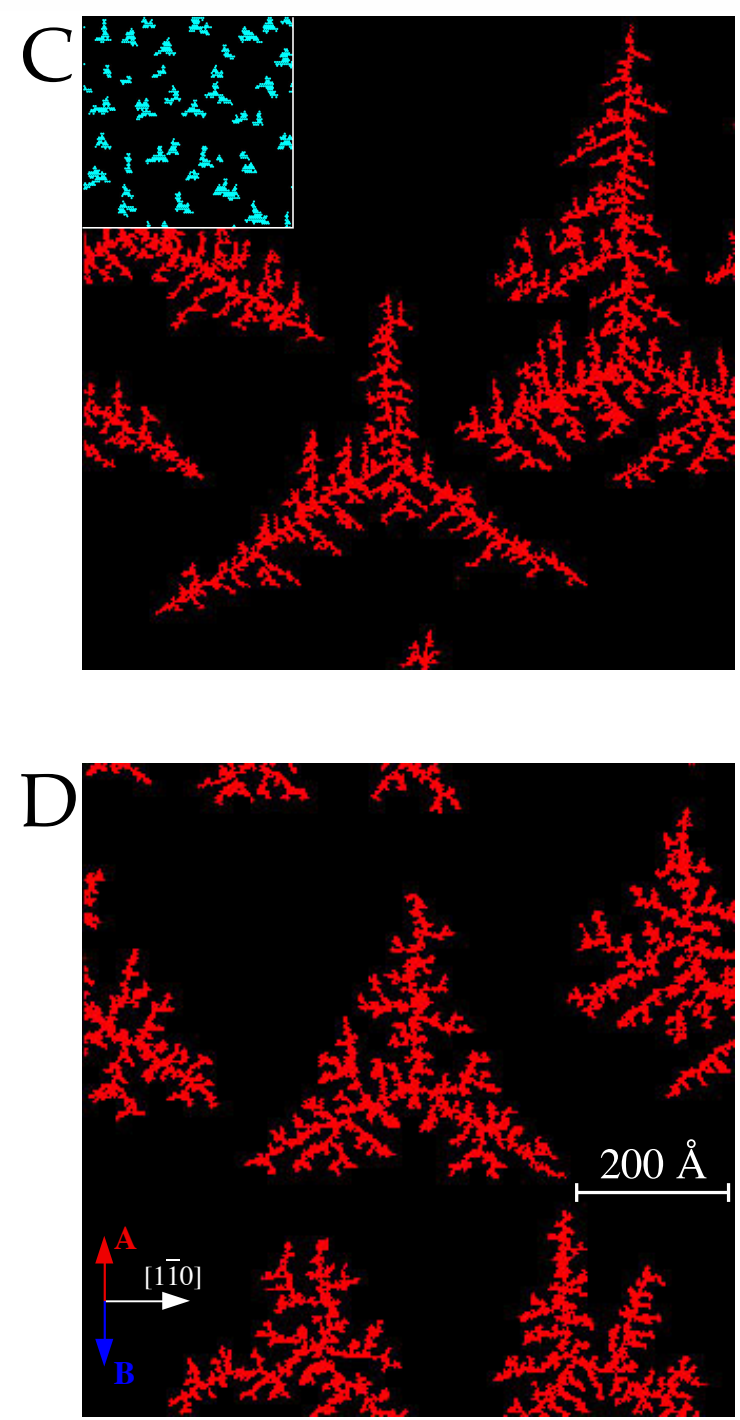

Fig. 4. KMC simulations of the STM experiments for $\mathrm{Ag} / \mathrm{Pt}(111)$. As in Fig. $1 \mathrm{~A}$, the scale is the same for figures and insets, they correspond to growth temperatures of 130 and $80 \mathrm{~K}$, respectively. Coverages and deposition rates are as in the experiment. For hit and stick, classical DLA fractals result (A). The key process for dendritic islands is diffusion from corner to A-steps (B). Close-packed chains that occasionally form in (B) are no more observed when a small atomic row is allowed to relax (C). Best agreement with experiment is obtained when corner to B-step diffusion is introduced (D). 


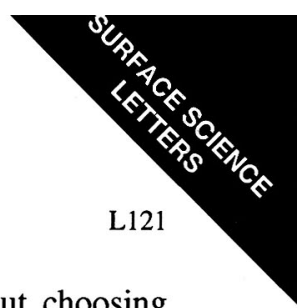

Therefore, growth of Y's occurs as A-steps effectively capture atoms, these atoms form tips which are traps for attaching atoms due to the frozen corner to $\mathrm{B}$ diffusion. Atoms attached there thus stay until a partner arrives to form a dimer which provides new A-steps. Direct attachment to B-steps leads to statistical deviations from this behavior. However, it also creates new A-steps which can be the origin for a new branch in the preferred A-directions.

To test these ideas we performed kinetic MonteCarlo (KMC) simulations [28] for $\mathrm{Ag} / \mathrm{Pt}(111)$ on a hexagonal lattice where we distinguish between Aand B-directions. Activation energies on surfaces are often underestimated by the EMT. However, the EMT generally gives a good idea on the energetic hierarchy of different diffusion processes. Therefore, as a first input for the KMC we use the EMT activation energies scaled by a common factor of 2 , since then the barrier for terrace diffusion equals the experimental value of $E_{\mathrm{m}}=160 \mathrm{meV}$ [39]. All prefactors have been set to the experimental value for terrace diffusion of $1 \times 10^{13} \mathrm{~s}^{-1}$ [39]. Using $E_{\mathrm{m}}=$ $160 \mathrm{meV}$ in a KMC simulation precisely reproduces the experimental island densities at temperatures up to $110 \mathrm{~K}$, where dimers are stable and immobile. In our simulations done at $130 \mathrm{~K}$, we have used $E_{\mathrm{m}}=$ $120 \mathrm{meV}$ as an effective barrier for terrace diffusion which then also accounts for the dimer instability or mobility. This way, we again get the experimental island density at $130 \mathrm{~K}$ and thus the correct lateral impinging rate of atoms to each island.

The KMC simulations nicely illustrate the influence of the different processes on the island shape (Fig. 4). The hit and stick case, where terrace diffusion and jump down from island tops are the only atomic displacements allowed, gives the classical randomly ramified DLA fractals where the substrate symmetry is not visible in the overall cluster shape (Fig. 4A). Turning on corner diffusion towards Asteps induces a marked change towards trigonal symmetry with branches predominantly growing into the A-direction (Fig. 4B). However the aggregates form monoatomic chains along $\langle 1 \overline{1} 0\rangle$ and the Y-shaped islands experimentally observed at $80 \mathrm{~K}$ cannot be reproduced (see inset Fig. 4B). If we introduce further the collective relaxation of these chains, we can improve this. The scaled EMT barrier of $E_{\text {coll }}=240$ $\mathrm{meV}$ (see Fig. 3B) is too high for the process to happen sufficiently frequent at $80 \mathrm{~K}$, but choosing $E_{\text {coll }}=160 \mathrm{meV}$ we get a strikingly good agreement with the experimental island shapes at 130 and $80 \mathrm{~K}$ (Fig. 4C and inset).

We want to point out that the important time scale for all relaxation processes at the island edge is the lateral impinging rate of diffusing atoms. The observed island shapes at $80 \mathrm{~K}$ set an upper limit of $160 \mathrm{meV}$ for the barriers of the corner to A process and the collective relaxation of the $\langle 1 \overline{1} 0\rangle$ chains. Speeding up these processes by reducing their energy barriers in the simulations would not significantly change the morphology of the obtained islands (Figs. 4B-4D). Similarly, we find that the experimentally observed island shapes at $130 \mathrm{~K}$ (Fig. 1A) set a lower limit of $320 \mathrm{meV}$ for the barrier of the corner to B process. The scaled EMT barrier of $500 \mathrm{meV}$ freezes this process completely even at 130 K. In Fig. 4D, we show the result of a simulation using $E_{\mathrm{Bc}}=320 \mathrm{meV}$ at $130 \mathrm{~K}$. This gives an even better agreement with the experimentally observed islands. There is still strongly preferred growth perpendicular to the A-steps, however, now the simulated islands bear some of the randomness characteristic of the experiment with occasional growth of the branches perpendicular to B-steps.

In general, the growth direction of the aggregate's branches is largely determined by corner diffusion. It decides whether randomly ramified or dendritic patterns will evolve, and determines the orientation of the latter. The branch width, on the other hand, is determined by edge diffusion, which has much less influence on their growth direction. Finally, compact islands can only be formed if atoms can leave the two-fold coordination at edges and diffuse around corners, a process which has a slightly higher barrier than edge diffusion (see Table 1).

In conclusion, we have presented STM experiments at low temperature showing dendritic growth for diffusion limited metal aggregation on three different hexagonal surfaces. The mechanism giving rise to the trigonal symmetry has been identified using EMT energy calculations and kinetic MonteCarlo simulations. The key process is the preferential diffusion of atoms from one-fold corner sites towards A-steps. In addition to this anisotropy in corner diffusion there is a preferred diffusion towards A-steps for atoms approaching an island. Both 


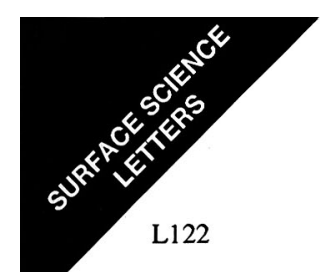

processes significantly increase the population of these steps and thereby promote trigonal dendritic growth.

\section{References}

[1] T.A. Witten and L.M. Sander, Phys. Rev. Lett. 47 (1981) 1400.

[2] T.A. Witten and L.M. Sander, Phys. Rev. B 27 (1983) 5686.

[3] P. Meakin, Phys. Rev. A 27 (1983) 1495.

[4] D. Grier, E. Ben-Jacob, R. Clarke and L.M. Sander, Phys. Rev. Lett. 56 (1986) 1264.

[5] Y. Sawada, A. Dougherty and J.P. Gollub, Phys. Rev. Lett. 56 (1986) 1260.

[6] M.B. Amer, P. Pelcé and P. Tabeling, Growth and Form: Nonlinear Aspects (Plenum, New York, 1991).

[7] H. Takayasu, Fractals in the Physical Sciences (Manchester University Press, Manchester, New York, 1990).

[8] P. Meakin, Phys. Rev. A 33 (1986) 3371.

[9] H. Brune, C. Romainczyk, H. Röder and K. Kern, Nature 369 (1994) 469.

[10] J. Nittmann and H.E. Stanley, J. Phys. A: Math. Gen. 20 (1987) L1185.

[11] T. Vicsek, Fractal Growth Phenomena (World Scientific, Signapore, 1989).

[12] P. Meakin, Phys. Rev. A 36 (1987) 332.

[13] D.D. Chambliss and R.J. Wilson, J. Vac. Sci. Technol. B 9 (1991) 928.

[14] A. Brodde, G. Wilhelmi, D. Badt, H. Wengelnik and H. Neddermeyer, J. Vac. Sci. Technol. B 9 (1991) 920

[15] R.Q. Hwang, J. Schröder, C. Günther and R.J. Behm, Phys. Rev. Lett. 67 (1991) 3279

[16] M.C. Bartelt and J.W. Evans, Surf. Sci. 314 (1994) L829.

[17] G.S. Bales and D.C. Chrzan, Phys. Rev. Lett. 74 (1995) 4879.

[18] A. Pimpinelli, J. Villain and D.E. Wolf, J. Phys. (Paris) 3 (1993) 447.
[19] H. Röder, K. Bromann, H. Brune and K. Kern, Phys. Rev. Lett. 74 (1995) 3217

[20] T. Michely, M. Hohage, M. Bott and G. Comsa, Phys. Rev. Lett. 70 (1993) 3943.

[21] H. Röder, E. Hahn, H. Brune, J.P. Bucher and K. Kern, Nature 366 (1993) 141.

[22] P. Jensen, A.L. Barabási, H. Larralde, S. Halvin and H.E. Stanley, Nature 368 (1994) 22.

[23] L. Bardotti, P. Jensen, A. Hoareau, M. Treilleux and B. Cabaud, Phys. Rev. Lett. 74 (1995) 4694.

[24] M.C. Bartelt and J.W. Evans, Phys. Rev. B 46 (1992) 12675.

[25] G.S. Bales and D.C. Chrzan, Phys. Rev. B 50 (1994) 6057.

[26] J.G. Amar and F. Family, Phys. Rev. Lett. 74 (1995) 2066.

[27] S. Liu, Z. Zhang, G. Comsa and H. Metiu, Phys. Rev. Lett. 71 (1993) 2967.

[28] J. Jacobsen, K.W. Jacobsen, P. Stoltze and J.K. Nørskov, Phys. Rev. Lett. 74 (1995) 2295.

[29] Z. Zhang, X. Chen and M.G. Lagally, Phys. Rev. Lett. 73 (1994) 1829.

[30] S.C. Wang and G. Ehrlich, Phys. Rev. Lett. 67 (1991) 2509.

[31] H. Brune, H. Röder, C. Boragno and K. Kern, Phys. Rev. B 49 (1994) 2997.

[32] H. Brune, H. Röder, C. Romainczyk, C. Boragno and K. Kern, Appl. Phys. A 60 (1995) 167.

[33] J. Kertész and T. Vicsek, J. Phys. A: Math. Gen. 19 (1986) L257.

[34] As came to our attention during preparation of this manuscript, Michely et al. suggest a similar explanation based on anisotropic corner diffusion.

[35] This important difference has first been realized by Zhang et al. in Ref. [29].

[36] In a former publication (Ref. [9]) we have suggested that anisotropy in edge diffusion might be responsible for dendritic growth.

[37] P. Stoltze, J. Phys. Condens. Matter 6 (1994) 9495.

[38] W.W. Mullins and R.F. Sekerka, J. Appl. Phys. A 34 (1963) 323.

[39] H. Brune, H. Röder, C. Boragno and K. Kern, Phys. Rev. Lett. 73 (1994) 1955. 